\title{
Tradeoff Analysis for Optimal Multiobjective Inventory Model
}

\author{
Longsheng Cheng, ${ }^{1}$ Ching-Shih Tsou, ${ }^{2}$ Ming-Chang Lee, ${ }^{3}$ Li-Hua Huang, ${ }^{4}$ \\ Dingwei Song, ${ }^{5}$ and Wei-Shan Teng ${ }^{2}$ \\ ${ }^{1}$ School of Economics and Management, Nanjing University of Science and Technology, Nanjing, Jiangsu 210094, China \\ ${ }^{2}$ Institute of Information and Decision Sciences, National Taipei College of Business, Taipei 10051, Taiwan \\ ${ }^{3}$ Department of Information Management, Yu Da University, Miaoli 36143, Taiwan \\ ${ }^{4}$ Department of Accounting Information, National Taipei College of Business, Taipei 10051, Taiwan \\ ${ }^{5}$ School of Management, Jiangsu University, Zhengjiang, Jiangsu 212013, China
}

Correspondence should be addressed to Ching-Shih Tsou; cstsou@mail.ntcb.edu.tw

Received 1 February 2013; Accepted 14 April 2013

Academic Editor: Farhad Hosseinzadeh Lotfi

Copyright (C) 2013 Longsheng Cheng et al. This is an open access article distributed under the Creative Commons Attribution License, which permits unrestricted use, distribution, and reproduction in any medium, provided the original work is properly cited.

Deterministic inventory model, the economic order quantity (EOQ), reveals that carrying inventory or ordering frequency follows a relation of tradeoff. For probabilistic demand, the tradeoff surface among annual order, expected inventory and shortage are useful because they quantify what the firm must pay in terms of ordering workload and inventory investment to meet the customer service desired. Based on a triobjective inventory model, this paper employs the successive approximation to obtain efficient control policies outlining tradeoffs among conflicting objectives. The nondominated solutions obtained by successive approximation are further used to plot a 3D scatterplot for exploring the relationships between objectives. Visualization of the tradeoffs displayed by the scatterplots justifies the computation effort done in the experiment, although several iterations needed to reach a nondominated solution make the solution procedure lengthy and tedious. Information elicited from the inverse relationships may help managers make deliberate inventory decisions. For the future work, developing an efficient and effective solution procedure for tradeoff analysis in multiobjective inventory management seems imperative.

\section{Introduction}

Inventory control is an important activity that appears in any kind of organization. For this reason, it has been studied extensively in the past several decades. Most inventory models aggregate different cost concepts, such as ordering cost, carrying cost, and shortage cost, into a single-objective formulation. Optimal decisions about when to order and how much to order are then solved by single-objective optimization techniques. However the insight gained from the oldest inventory model, economic order quantity (EOQ), reveals that inventory management should be considered as a biobjective optimization problem to strike a balance between inventory carrying and annual orders. Practically speaking, inventory decisions involve tradeoffs related to operational efficiency and customer service.
Brown $[1,2]$ first examined the tradeoff between investment in working stock and annual ordering cost. He introduced the concept of exchange curve shown in Figure 1. The curve demonstrates how capital invested in working stocks can be traded for operating expenses of ordering. Points below the curve are infeasible, and decisions located above the curve are suboptimal. Suboptimal policies can be improved by moving back to the curve (i.e., seeking possible improvement from point $\mathrm{A}$ to $\mathrm{B}$ or $\mathrm{C})$. Starr and Miller [3] determined tradeoffs between two performance measures: (i) number of orders per year (workload) and (ii) average investment in inventory in the case of multiple items. Gardner and Dannenbring [4] introduced customer service as another measure, along with workload and inventory investment, and generalized above exchange curve analysis to the optimal policy surface in case of probabilistic demand. 


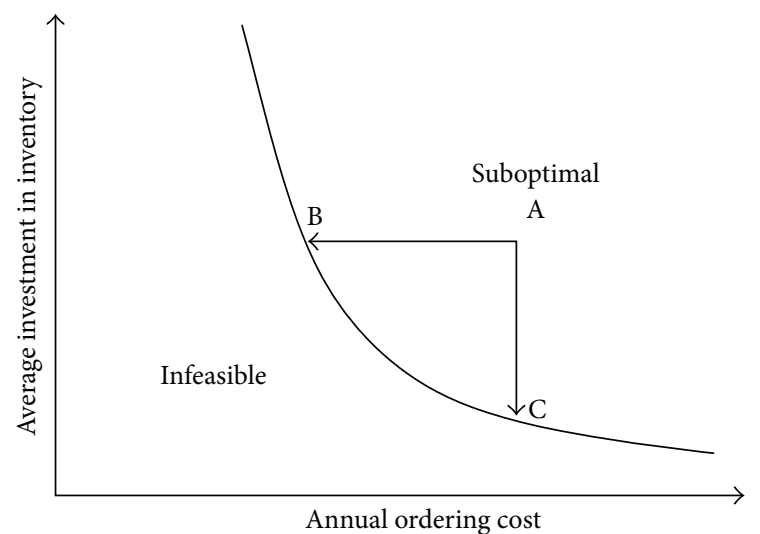

FIgURE 1: The exchange curve for deterministic inventory model.

The model and solution technique they used is still based on single-objective optimization. Bookbinder and Chen [5] proposed a multiobjective formulation for analyzing multiechelon inventory and distribution systems. They argued that points on exchange curve or policy surface are equivalent to the nondominated solutions concept of multiobjective optimization. Although it could be the first multiple criteria generalizations of earlier studies, the model was solved by classical optimization techniques.

To a certain extent, the aforementioned tradeoff analysis of inventory management is developed by single-objective optimization. The motivation of this study aims to develop an intrinsically multiobjective approach for building the tradeoff surface of probabilistic inventory systems. Differences between traditional and multiobjective approach are not only in their problem formulations, but also the latter simultaneously treats several objectives analytically or heuristically under certain notion of multiobjective optimality $[6,7]$.

Gutiérrez et al. [8] considered a dynamic single facility single-item lot size problem. Although the total demand is assumed to be a fixed value, the distribution of this demand among different periods is unknown. They determined all the Pareto-optimal or nondominated production plans that are robust to any possible occurrence of all scenarios. Gutiérrez et al. [9] presented the characterization of the nondominated optimal solution set and use it to correct the solution method previously proposed by Bookbinder and Chen [5].

For the multiobjective exchange curve, Tsou [7] presented a two-stage framework consisting of multiobjective particle swarm optimization (MOPSO) and technique for order preference by similarity to ideal solution (TOPSIS). At the first stage, MOPSO is used to generate the tradeoff (or nondominated) front of the triobjective inventory model in Agrell [10]. Then, a preferred solution is selected by TOPSIS according to subjective preferences of decision makers. Tsou and Kao [11] also developed a metaheuristic based on electromagnetism-like mechanism (EM) to approximate the Pareto-optimal front without using any prior or interactive preference. They showed that the metaheuristic can find similar Pareto-optimal solutions as the popular interactive procedure Step method (STEM) did [12]. Tsou [13] further showed that evolutionary Pareto optimizers could generate tradeoff solutions potentially ignored by the well-known simultaneous method.

Nevertheless, we recently notice that the tradeoff solutions of the above studies actually laid on an exchange curve, instead of forming a tradeoff surface in the $3 \mathrm{D}$ objective space. It apparently indicates that some of the objectives, including minimization of expected annual cost, expected annual number of stockout occasions, and expected annual number of items stocked out, are not conflicting with each other. Among which, the last two objectives are redundant because they relate to the same concept of shortage but different measures. Consequently, such a kind of triobjective models was not properly justified in the above studies.

This paper first presents a triobjective model without redundancy in the next section. Nonredundancy is assured by dropping all the marginal cost parameters out of the classical fixed order model. After that, a successive approximation procedure based on the Lagrange method is utilized to iteratively search for nondominated solutions and efficient control policies. Tradeoffs among workload, inventory, and shortage are visualized by three-dimensional scatterplots. Although it is a time-consuming job to use the successive approximation to find the tradeoff surfaces of multiobjective model, all solutions found are ensured to be Pareto-optimal in comparison with other search methods, such as genetic algorithms. Finally, conclusions and directions for future research are drawn out accordingly.

\section{Model Building and Solution Procedures}

2.1. A Triobjective Model. The reorder point lot size system, $(r, Q)$, is a popular control method under probabilistic demand. An order of size $Q$ will be triggered immediately whenever the inventory position drops to the reorder point $r$ or lower. Classical $(r, Q)$ model minimizes a lump-sum cost including ordering cost, carrying cost, and stockout cost [14]. The triobjective model below intrinsically restores the nature of conflicts among objectives that are to minimize the workload, inventory, and shortage. Also, multiobjective $(r, Q)$ model does not run into the incommensurate issue while aggregating objectives of different measures into a single one. The notations used here are described as follows.

$D$ is the average annual demand,

$L$ is the lead time,

$D_{L}$ is the lead time demand. It is normally distributed with mean $\mu_{L}$ and standard deviation $\sigma_{L}$,

$S S$ is the safety stock, which is proportional to the standard deviation of lead time demand. That is, $S S=$ $k \sigma_{L}$, where $k$ represents the safety factor,

$r$ is the aforementioned reorder point, which equals to the average lead time demand plus the safety stock. That is, $r=\mu_{L}+k \sigma_{L}$, and $\varphi(z)$ is the probability density function of standard normal distribution. 
A multiobjective $(r, Q)$ model is formulated as follows:

$$
\begin{array}{ll}
\underset{k, \mathrm{Q}}{\operatorname{Min}} & W=\frac{D}{\mathrm{Q}}, \\
\operatorname{Min}_{k, \mathrm{Q}} & I=\frac{\mathrm{Q}}{2}+k \sigma_{L}, \\
\underset{k, \mathrm{Q}}{\operatorname{Min}} & S=\frac{D \sigma_{L}}{\mathrm{Q}} \int_{k}^{\infty}(z-k) \varphi(z) d z
\end{array}
$$

subject to the following:

$$
\begin{aligned}
& 0 \leq Q \leq D, \\
& 0 \leq k \leq \frac{D}{\sigma} .
\end{aligned}
$$

Equation (1) represents the number of annual order (in cycles per year, also called workload). Equation (2) is the sum of cycle and safety stocks (in units carrying per year). Equation (3) denotes the average number of demand not covered from stock annually (in units short per year). Inequality (4) ensures that the order size (units per order) should be nonnegative and not more than the average annual demand. Inequality (5) guarantees that the safety stock (in units) will not be greater than the average annual demand and should be nonnegative.

The notion of optimality in single-objective optimization is straightforward, because the optimal solution is the one that realizes the maximum (or the minimum) of the objective function. However, the optimality for a multiobjective optimization problem is not so easy to understand because not all feasible solutions can be compared completely. Generally speaking, multiobjective optimization problems rarely have solutions that simultaneously optimize all of the objectives; as a result we are trying to optimize each objective to the greatest extent possible. There exists a set of solutions, referred as nondominated solutions, which are better than others in the search space when considering all the objectives. For the minimization problem (in Section 2.1), a control parameter $x^{1}=\left(k_{1}, Q_{1}\right)$ is said to strongly dominate $x^{2}=\left(k_{2}, Q_{2}\right)$ (denoted by $x^{1}<x^{2}$ ) if and only if $W\left(x^{1}\right)<W\left(x^{2}\right), I\left(x^{1}\right)<$ $I\left(x^{2}\right)$, and $S\left(x^{1}\right)<S\left(x^{2}\right)$. That is, solution $x^{1}$ is strictly better than solution $x^{2}$ in all the cost and service objectives ([15, pp. 32]). Less stringently, a decision vector $x^{1}$ dominates $x^{2}$ (denoted by $x^{1} \leq x^{2}$ ) if and only if $W\left(x^{1}\right) \leq W\left(x^{2}\right), I\left(x^{1}\right) \leq$ $I\left(x^{2}\right), S\left(x^{1}\right) \leq S\left(x^{2}\right)$ and at least one of above inequality is strictly held ([15, pp. 28]). For other multiobjective (or multicriteria) concepts, please refer to Ehrgott [16].

Undoubtedly, we are not interested in solutions dominated by other solutions. Solutions that are not dominated by any other solutions are called nondominated in objective space or efficient in decision space. It means that the improvement of some objective could only be achieved at the expense of other objectives. This coincides with the exchange curve concept mentioned earlier. In a multiobjective optimization problem, there are normally a large number of nondominated solutions due to the conflicts among objectives. Hence, it is difficult to find the whole set of nondominated solutions.
And because the nondominated set is usually unknown, most optimizers try to find a finite number of nondominated solutions to approximate the set. The successive approximation approach stated below can be used to search for the nondominated solutions of the triobjective inventory model.

2.2. Successive Approximation Based on Lagrange Method. A single objective transformation is first developed as follows. Equation (3) is kept as the objective function and treats (1) and (2) as constraints. That is,

$$
\begin{array}{ll}
\underset{k, Q}{\operatorname{Min}} S & \\
\text { subject to } & W=W^{\prime} \\
& I=I^{\prime},
\end{array}
$$

where $W^{\prime}$ and $I^{\prime}$ are budgets on workload and inventory.

To solve this equality constrained optimization problem, the Lagrange method is employed here. After introducing the Lagrangian multipliers $\lambda_{W}$ and $\lambda_{I}$, the Lagrangian function is as follows:

$$
\begin{aligned}
L\left(k, Q, \lambda_{W}, \lambda_{I}\right)= & \frac{D \sigma_{L}}{Q} \int_{k}^{\infty}(z-k) \varphi(z) d z \\
& +\lambda_{I}\left(\frac{Q}{2}+k \sigma_{L}-I^{\prime}\right)+\lambda_{W}\left(\frac{D}{Q}-W^{\prime}\right) .
\end{aligned}
$$

Some simplifying notations are introduced before presenting the successive approximation algorithm. Let

$$
\begin{gathered}
P=\int_{k}^{\infty} \varphi(z) d z, \\
E=\sigma_{L} \int_{k}^{\infty}(z-k) \varphi(z) d z,
\end{gathered}
$$

$P$ is the probability of a stockout per order cycle and $E$ is the expected number of shortage per order cycle. Simple algebra provides the following equations used in the successive approximation:

$$
\begin{gathered}
\lambda_{I}=\frac{D P}{2\left(I^{\prime}-k \sigma\right)}, \\
P=\frac{\lambda_{I} Q}{D}, \\
\lambda_{W}=\frac{1}{W^{\prime}}\left(\frac{\lambda_{I} Q}{2}-\frac{D E}{Q}\right), \\
Q=\sqrt{\frac{2 D\left(E+\lambda_{W}\right)}{\lambda_{I}} .}
\end{gathered}
$$

To search for the efficient $\left(k_{i}, Q_{i}\right)$ policy, the search steps are proposed as follows. 


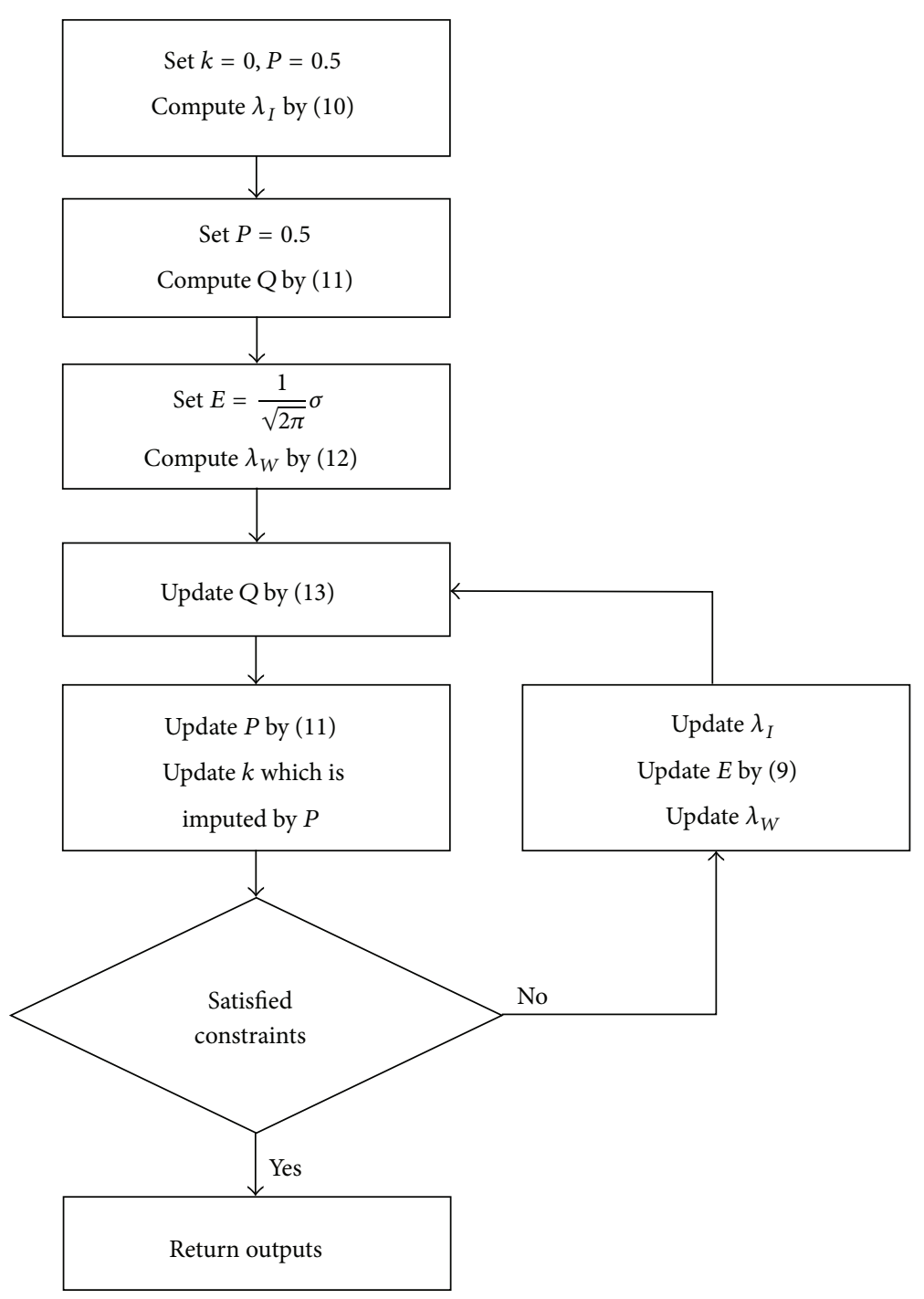

Figure 2: Flow chart of the search algorithm.

Step 1. Initialize $\lambda_{I}$ : Compute $\lambda_{I}$ using (10) with $k=0$ and $P=0.5$.

Step 2. Initialize $Q$ : Compute $Q$ using (11) with $P=0.5$.

Step 3. Initialize $\lambda_{W}$ : Compute $\lambda_{W}$ using (12) with $E=$ $(1 / \sqrt{2 \pi}) \sigma$ corresponds to zero safety stock.

Step 4. Update Q: Compute Q using (13).

Step 5. Update $P$ and $k$ : Compute $P$ with (11) and look up $k$ imputed by $P$.

Step 6. Check constraints: If both workload and investment constraints are satisfied, then output the results. Otherwise, update $\lambda_{I}, E$ (using (9)), and $\lambda_{W}$, then go back to Step 4 .

The search begins with an initial guess of zero safety stock. This allows us to use (10) to derive an initial $\lambda_{I}$, which in turn, determines the initial $Q$ using (11). However, (12)-(13) for $\lambda_{W}$ and $Q$ are interdependent, preventing their use in the initialization phase. Rearranging (11), however, we can derive an equation for $Q$ which does not require an estimate of $\lambda_{W}$. The $Q$ based on (11) is then used to provide an initial estimate of $\lambda_{W}$ from (13). Thereafter the search progresses by iteratively updating values for $Q, P$ (and correspondingly $k$ ), $\lambda_{I}$, and $\lambda_{W}$, using (13), (11), (10), and (12), respectively, until both the workload and investment constraints are satisfied. The flow chart of the above search algorithm is shown in Figure 2.

\section{Numerical Results}

Pharmaceutical inventory data with four items (Table 1) were fed into the triobjective model. The successive approximation was coded in R [17], and all computation was executed on a laptop computer. Ten representative solutions for each 
TABLE 1: The pharmaceutical data.

\begin{tabular}{lcc}
\hline Item & $D$ & $\sigma_{L}$ \\
\hline 1 & 3412 & 53.354 \\
2 & 490 & 5.027 \\
3 & 4736 & 57.911 \\
4 & 200 & 2.969 \\
\hline
\end{tabular}

TABLE 2: Tradeoff solutions of Item 1 generated by successive approximation.

\begin{tabular}{lcccccc}
\hline \multirow{2}{*}{ Sol. no. } & \multicolumn{2}{c}{ Efficient solution } & \multicolumn{4}{c}{ Nondominated solution } \\
& $Q$ & $k$ & $W$ & $I$ & $S$ & Iter \\
\hline 1 & 94.804 & 0.049 & 35.990 & 50.000 & 720.207 & $81^{\star}$ \\
2 & 162.532 & 0.351 & 20.993 & 99.992 & 277.522 & $20^{\dagger}$ \\
3 & 98.804 & 0.986 & 35.990 & 99.999 & 164.354 & 34 \\
4 & 83.238 & 1.094 & 40.991 & 99.999 & 151.799 & 41 \\
5 & 162.51 & 1.287 & 20.996 & 149.931 & 52.407 & $20^{\dagger}$ \\
6 & 94.798 & 1.923 & 35.992 & 149.993 & 19.999 & 27 \\
7 & 131.234 & 2.517 & 25.999 & 199.92 & 2.635 & 26 \\
8 & 213.250 & 2.686 & 16.000 & 249.915 & 0.948 & 41 \\
9 & 110.065 & 3.653 & 31.000 & 249.922 & 0.052 & 32 \\
10 & 110.065 & 4.590 & 31.000 & 299.911 & 0.001 & 40 \\
Min. & 83.238 & 0.049 & 16.000 & 50.000 & 0.001 & 20 \\
Max. & 213.250 & 4.590 & 40.991 & 299.911 & 720.207 & 81 \\
\hline
\end{tabular}

${ }^{\dagger}$ and ${ }^{*}$ represent the minimum and the maximum numbers of iterations, respectively.

item generated by successive approximation are shown in Tables 2, 3, 4, and 5 . The columns of nondominated solutions demonstrate that the improvement of some objective(s) could only be achieved at the expense of other objectives. For example, solution 3, compared to solution 4, in Table 2 gets better in workload at the expense of shortage.

Three-dimensional scatterplots for each item are illustrated in Figures 3, 4, 5, and 6. Any one can visually check the tradeoffs displayed in scatterplots by adding planes parallel to $I-S$ or $W-S$ plane. With a fixed workload, expected shortage decreases as expected inventory increases. At a fixed inventory level, increases in workload lead to a reduction of expected shortage. All these findings are intuitive and straightforward.

For the computation effort, we notice that only one solution in Table 2 can be obtained after at least twenty iterations. And the largest iterations to reach a nondominated solution is eighty-one. Ranges of other items are between eight to sixty-six iterations. Hence, creating the scatterplot of workload, inventory, and shortage by successive approximation is lengthy and tedious.

The quality of a set of tradeoff solutions is evaluated quantitatively in terms of accuracy and diversity. A metric called hypervolume $(H)$ is used to demonstrate the accuracy of the nondominated solutions. It calculates the size of the area that is dominated by a nondominated set and is defined as follows. The idea is that the larger the area the solutions can
TABLE 3: Tradeoff solutions of Item 2 generated by successive approximation.

\begin{tabular}{lcccccc}
\hline \multirow{2}{*}{ Sol. no. } & \multicolumn{2}{c}{ Efficient solution } & \multicolumn{4}{c}{ Nondominated solution } \\
& $Q$ & $k$ & $W$ & $I$ & $S$ & Iter \\
\hline 1 & 18.8519 & 0.114 & 25.9921 & 9.9989 & 45.0181 & $15^{\dagger}$ \\
2 & 23.3406 & 0.6575 & 20.9935 & 14.9757 & 16.193 & $15^{\dagger}$ \\
3 & 30.6264 & 0.9159 & 15.9992 & 19.9176 & 7.8443 & 17 \\
4 & 13.6139 & 1.6284 & 35.9927 & 14.9929 & 3.9316 & 21 \\
5 & 11.9541 & 1.794 & 40.9901 & 14.9954 & 2.9865 & 22 \\
6 & 18.8469 & 2.0863 & 25.999 & 19.9113 & 0.8779 & 21 \\
7 & 15.8077 & 2.3897 & 30.9976 & 19.917 & 0.4372 & 21 \\
8 & 11.9532 & 2.781 & 40.9934 & 19.9568 & 0.1671 & 22 \\
9 & 18.8462 & 3.0794 & 26 & 24.9033 & 0.0376 & 30 \\
10 & 30.625 & 3.8973 & 16 & 34.9042 & 0.0009 & $60^{\star}$ \\
Min. & 11.9532 & 0.114 & 15.9992 & 9.9989 & 0.0009 & 15 \\
Max. & 30.6264 & 3.8973 & 40.9934 & 34.9042 & 45.0181 & 60 \\
\hline
\end{tabular}

TABLE 4: Tradeoff solutions of Item 3 generated by successive approximation.

\begin{tabular}{lcccccc}
\hline \multirow{2}{*}{ Sol. no. } & \multicolumn{2}{c}{ Efficient solution } & \multicolumn{4}{c}{ Nondominated solution } \\
& $Q$ & $k$ & $W$ & $I$ & $S$ & Iter \\
\hline 1 & 296.134 & 0.032 & 15.993 & 149.924 & 354.824 & $8^{\dagger}$ \\
2 & 430.546 & 0.598 & 11 & 249.913 & 107.77 & 25 \\
3 & 789.333 & 0.954 & 6 & 449.91 & 31.58 & $59^{\star}$ \\
4 & 152.82 & 1.27 & 30.991 & 149.964 & 87.038 & 21 \\
5 & 225.525 & 1.505 & 21 & 199.918 & 35.237 & 25 \\
6 & 152.781 & 2.133 & 30.999 & 199.911 & 10.597 & 24 \\
7 & 115.534 & 2.455 & 40.992 & 199.945 & 5.463 & 24 \\
8 & 296 & 2.623 & 16 & 299.901 & 1.26 & 50 \\
9 & 131.556 & 3.18 & 36 & 249.913 & 0.416 & 30 \\
10 & 152.774 & 3.86 & 31 & 299.91 & 0.024 & 41 \\
Min. & 115.512 & 0.032 & 6 & 149.924 & 0.024 & 8 \\
Max. & 789.333 & 3.86 & 40.992 & 499.91 & 354.824 & 59 \\
\hline
\end{tabular}

dominate in the objective space, the better it is [18]:

$$
\prod_{i=1}^{M}\left(f_{i}^{\max }-f_{i}^{\min }\right)
$$

where $M$ is the number of objectives. Figure 7 shows the pictorial explanation of $H$ in which $O^{\prime}$ represents the reference point and $S$ is the nondominated set.

Keeping the nondominated set as diverse as possible is very important. Here spacing $(S)$ and maximum spread (D) are used to evaluate the distribution and spread of 
TABLE 5: Tradeoff solutions of Item 4 generated by successive approximation.

\begin{tabular}{lcccccc}
\hline \multirow{2}{*}{ Sol. no. } & \multicolumn{2}{c}{ Efficient solution } & \multicolumn{4}{c}{ Nondominated solution } \\
& $Q$ & $k$ & $W$ & $I$ & $S$ & Iter \\
\hline 1 & 18.1965 & 0.2896 & 10.9911 & 9.9582 & 8.8351 & $10^{\dagger}$ \\
2 & 5.5568 & 0.7482 & 35.9916 & 5 & 14.059 & 36 \\
3 & 33.3333 & 1.0901 & 6 & 19.9032 & 1.2465 & 28 \\
4 & 12.5064 & 1.2415 & 15.9918 & 9.9393 & 2.4446 & 15 \\
5 & 9.527 & 1.7531 & 20.993 & 9.9686 & 1.0003 & 18 \\
6 & 7.6947 & 2.0674 & 25.9918 & 9.9854 & 0.546 & 20 \\
7 & 5.5571 & 2.4315 & 35.9902 & 9.9978 & 0.2642 & 24 \\
8 & 33.3333 & 2.7747 & 6 & 24.9047 & 0.0148 & $66^{\star}$ \\
9 & 12.5 & 2.9139 & 16 & 14.9015 & 0.0245 & 28 \\
10 & 7.6925 & 3.7241 & 25.9993 & 14.9032 & 0.0018 & 25 \\
Min. & 5.5568 & 0.2896 & 6 & 5 & 0.0018 & 10 \\
Max. & 33.3333 & 3.7241 & 35.9916 & 24.9047 & 14.059 & 66 \\
\hline
\end{tabular}

${ }^{\dagger}$ and ${ }^{*}$ represent the minimum and the maximum numbers of iterations, respectively.

the nondominated fronts generated by successive approximation:

$$
\begin{gathered}
S=\sqrt{\frac{1}{|\widetilde{A}|} \sum_{i=1}^{|\widetilde{A}|}\left(d_{i}-\bar{d}\right)^{2}}, \\
D=\sqrt{\sum_{k=1}^{3}\left(\max _{i=1}^{|\widetilde{A}|} f_{k}^{i}-\min _{i=1}^{|\widetilde{A}|} f_{k}^{i}\right)^{2}},
\end{gathered}
$$

where $d_{i}=\min _{j \in \widetilde{A} \wedge j \neq i} \sum_{k=1}^{3}\left|f_{k}^{i}-f_{k}^{j}\right|, f_{k}^{i}$ represents the $k$ th criterion function value of the nondominated solution $i$, and $\bar{d}$ is the mean value of the absolute distance measure where $\bar{d}=\sum_{i=1}^{|\widetilde{A}|}\left(d_{i} /|\widetilde{A}|\right)$.

Larger above measures are better except for the spacing. Table 6 shows the results of the successive approximation method. If there is a reference solutions set known to decision makers or generated by other solution procedures, one can use the figures in Table 6 to compare successive approximation with the benchmark that he/she is interested in. After verifying the validity of nondominated $(W, I, S)$ solutions generated by successive approximation, they can be used to construct a tradeoff surface for inventory control. It helps managers choose an appropriate control policy for probabilistic demand, such as the fund level tied in inventory versus the service level under fixed workload.

\section{Conclusions and Suggestions}

Tradeoff analysis in inventory management is useful in quantifying what the firm must pay in terms of workload and investment to meet the desired customer service. A triobjective model is presented first to generate the efficient $(k, Q)$ policies in decision space that correspond to the nondominated $(W, I, S)$ solutions in objective space. Nonredundancy is assured by dropping all the marginal cost parameters out of the classical fixed order model. Such that

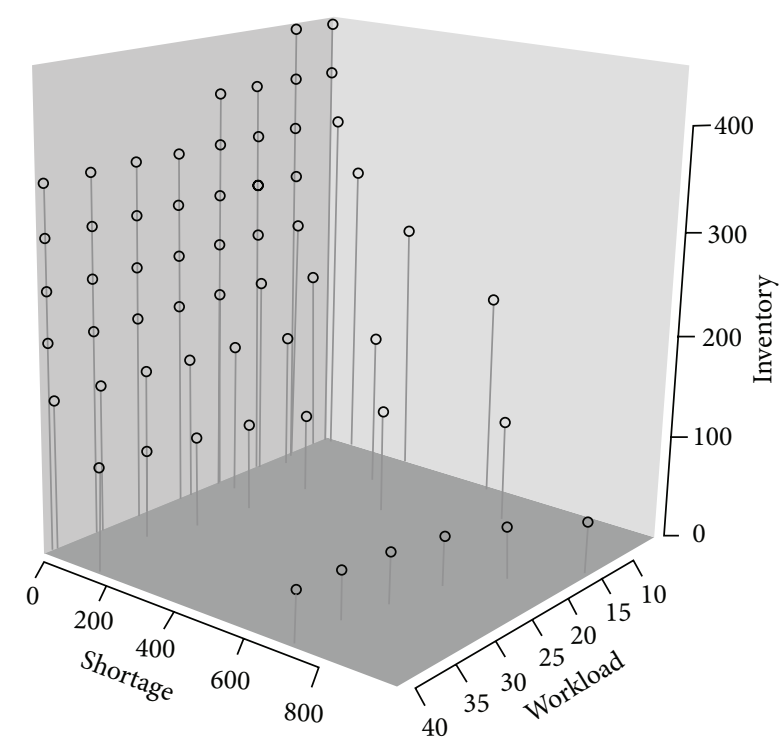

FIgURE 3: Scatterplot of Item 1 nondominated solutions from successive approximation.

TABle 6: Performance measures of the successive approximation approach.

\begin{tabular}{lccc}
\hline \multirow{2}{*}{ ID } & Accuracy & \multicolumn{2}{c}{ Distribution } \\
& $H$ & Spacing & Spread \\
\hline 1 & $42,694,515$ & 0.091 & 1.532 \\
2 & $7,215,908$ & 0.097 & 1.232 \\
3 & $68,798,878$ & 0.121 & 1.637 \\
4 & 476,041 & 0.105 & 1.362 \\
\hline
\end{tabular}

leads to a triobjective model which intends to minimize the workload, inventory, and shortage that are all conflicting with each other.

To solve the triobjective model, successive approximation approach is employed in this paper. The successive approximation is in an attempt to build the optimal tradeoff surface under probabilistic demand. Successive approximation can be used to obtain the nondominated solutions of workload, inventory, and shortage. Specifically, lots of trial and error involve in deriving the edge of each objective with a Lagrangian model. Several iterations needed to reach a nondominated solution for all items make the creating of scatterplot of workload, inventory, and shortage by successive approximation lengthy and tedious. However, visualization of the tradeoffs displayed by the scatterplots of Figures 3, 4, 5, and 6 justifies the computation effort done in the experiment. The inverse relationship among workload, inventory, and shortage conforms to our intuition.

The quality of a set of tradeoff solutions has to be evaluated quantitatively when comparing to other benchmarks, although developing an efficient and effective solution procedure for tradeoff analysis of multiobjective inventory management will be our future work. After verifying the validity of nondominated $(W, I, S)$ solutions, they can be used to construct a tradeoff surface that helps managers choose 


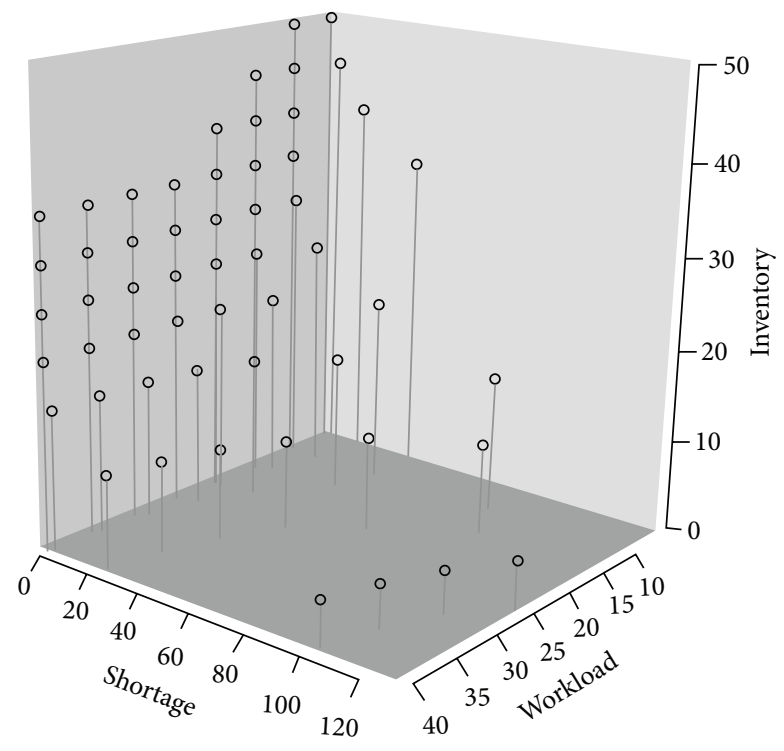

FIGURE 4: Scatterplot of Item 2 nondominated solutions from successive approximation.

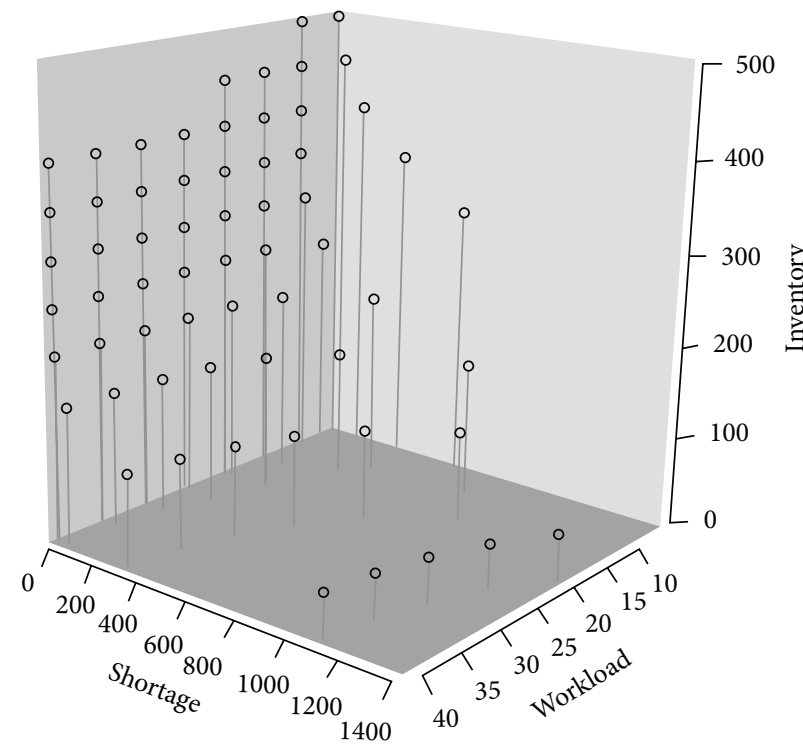

FIGURE 5: Scatterplot of Item 3 nondominated solutions from successive approximation.

an appropriate control policy for probabilistic demand. For example, a common debate on the fund level tied in inventory versus the service level under fixed workload usually arises between warehousing and sales departments. By utilizing the information coming from the tradeoff surface, deliberate decisions among conflicting objectives can be easily made.

Moreover, the tradeoff surface under multi-item context deserves closer attention to bridge the gap between inventory theory and managerial practice, because too much attention was focused on the single-item problem in view of the past literature. Finally, multiechelon inventory and/or

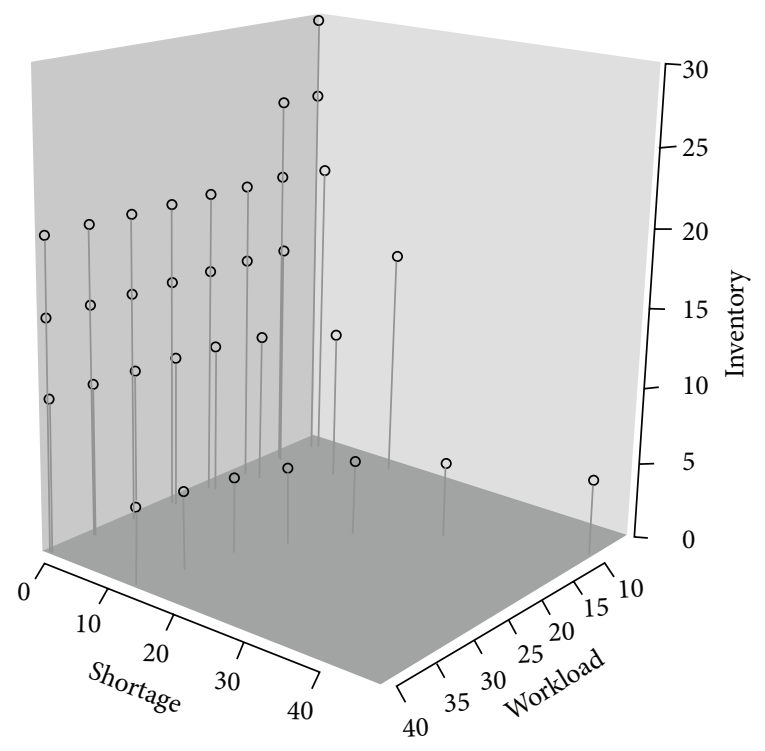

FIgURE 6: Scatterplot of Item 4 nondominated solutions from successive approximation.

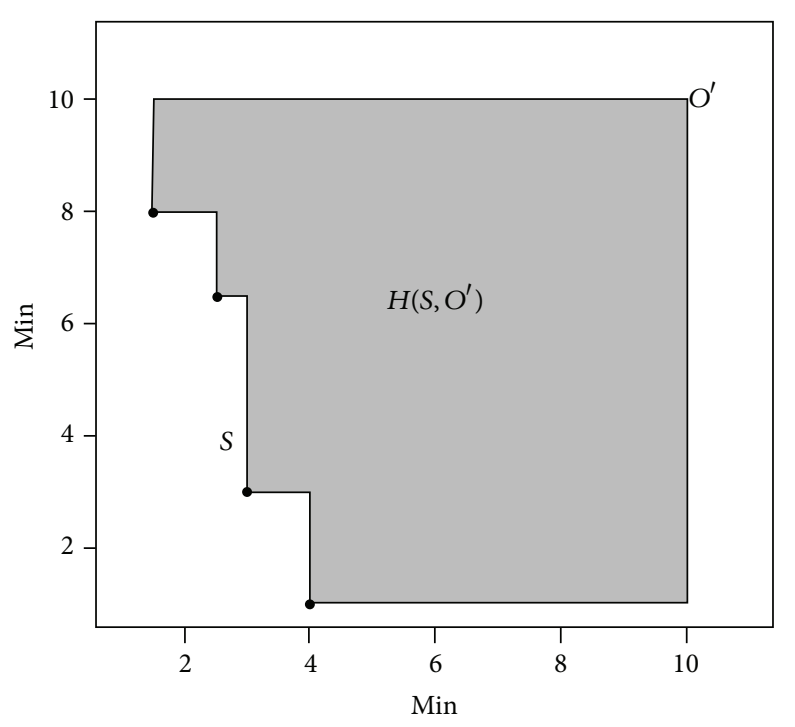

FIgURE 7: The hypervolume index in the minimization problem.

distribution systems are very common in business logistics. It is worthwhile to study the multiobjective inventory policies of different parties in a supply chain.

\section{Acknowledgments}

The authors would like to express special thanks to the National Science Council of Taiwan for financial support in NSC100-2628-H-141-001-MY2. This research was also partially supported by the National Natural Science Foundation of China (Grant 71271114). 


\section{References}

[1] R. G. Brown, "Use of carrying charge to control cycle stocks," APICS Quarterly Bulletin, vol. 2, pp. 29-46, 1961.

[2] R. G. Brown, Decision Rules for Inventory Management, Dryden Press, Illinois, Ill, USA, 1967.

[3] M. K. Starr and D. W. Miller, Inventory Control: Theory and Practice, Prentice Hall, New Jersey, NJ, USA, 1962.

[4] E. S. Gardner and D. G. Dannenbring, "Using optimal policy surfaces to analyze aggregate inventory tradeoffs," Management Science, vol. 25, no. 8, pp. 709-720, 1979.

[5] J. Bookbinder and V. Y. X. Chen, "Multicriteria trade-offs in a warehouse/retailer system," Journal of the Operational Research Society, vol. 43, no. 7, pp. 707-720, 1992.

[6] J. Puerto and F. R. Fernández, "Pareto-optimality in classical inventory problems," Naval Research Logistics, vol. 45, no. 1, pp. 83-98, 1998.

[7] C. S. Tsou, "Multi-objective inventory planning using MOPSO and TOPSIS," Expert Systems with Applications, vol. 35, no. 1-2, pp. 136-142, 2008.

[8] J. Gutiérrez, J. Puerto, and J. Sicilia, "The multiscenario lot size problem with concave costs," European Journal of Operational Research, vol. 156, no. 1, pp. 162-182, 2004.

[9] J. Gutiérrez, J. Puerto, and J. Sicilia, "Determining the Pareto set in a bicriteria two-echelon inventory/distribution system," Optimization, vol. 59, no. 2, pp. 253-271, 2010.

[10] P. J. Agrell, "A multicriteria framework for inventory control," International Journal of Production Economics, vol. 41, no. 1-3, pp. 59-70, 1995.

[11] C. S. Tsou and C. H. Kao, "Multi-objective inventory control using electromagnetism-like meta-heuristic," International Journal of Production Research, vol. 46, no. 14, pp. 3859-3874, 2008.

[12] R. Benayoun, J. de Montgolfier, J. Tergny, and O. Laritchev, "Linear programming with multiple objective functions: step method (stem)," Mathematical Programming, vol. 1, no. 1, pp. 366-375, 1971.

[13] C. S. Tsou, "Evolutionary Pareto optimizers for continuous review stochastic inventory systems," European Journal of Operational Research, vol. 195, no. 2, pp. 364-371, 2009.

[14] E. A. Silver, D. F. Pyke, and R. Peterson, Inventory Management and Production Planning and Scheduling, John Wiley \& Sons, New York, NY, USA, 1998.

[15] K. Deb, Multi-Objective Optimization Using Evolutionary Algorithms, John Wiley \& Sons, Chichester, UK, 2001.

[16] M. Ehrgott, Multicriteria Optimization, Springer, Berlin, Germany, 2nd edition, 2005.

[17] R Development Core Team, R: A Language and Environment for Statistical Computing, R Foundation for Statistical Computing, Vienna, Austria, 2009, http://www.r-project.org/.

[18] T. Okabe, Y. Jin, and B. Sendhoff, "A critical survey of performance indices for multi-objective optimization," in Proceedings of the IEEE Congress on Evolutionary Computation, vol. 2, pp. 878-885, IEEE Press, New Jersey, NJ, USA, 2003. 


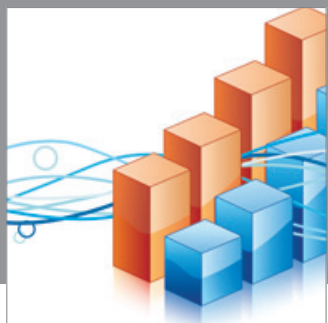

Advances in

Operations Research

mansans

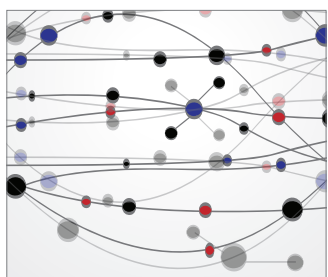

The Scientific World Journal
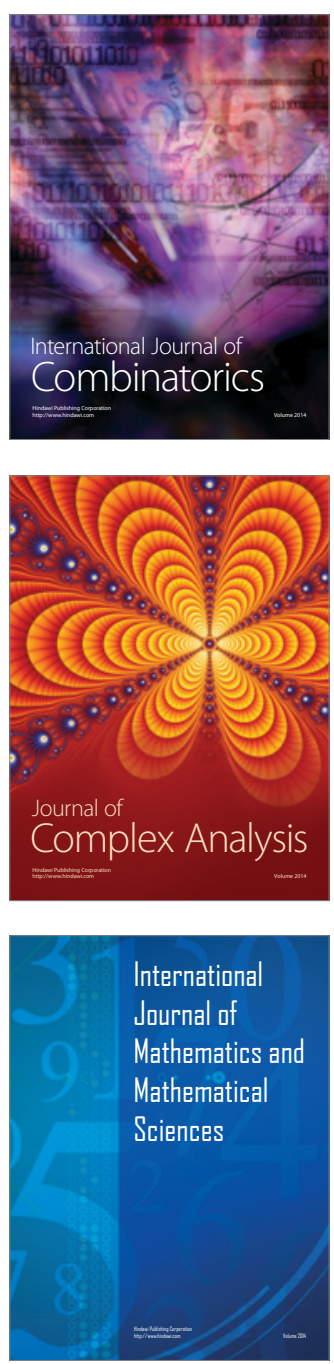
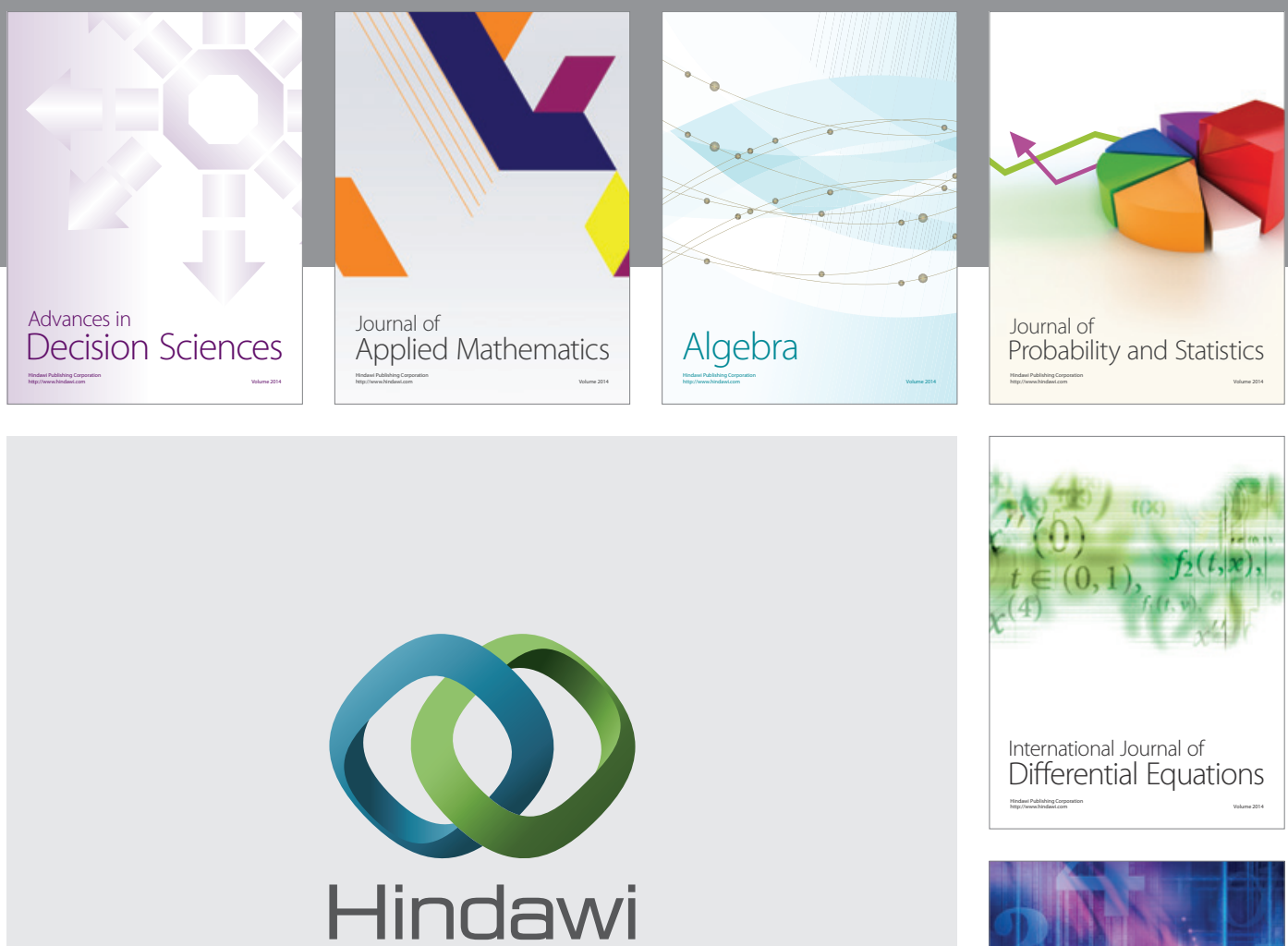

Submit your manuscripts at http://www.hindawi.com
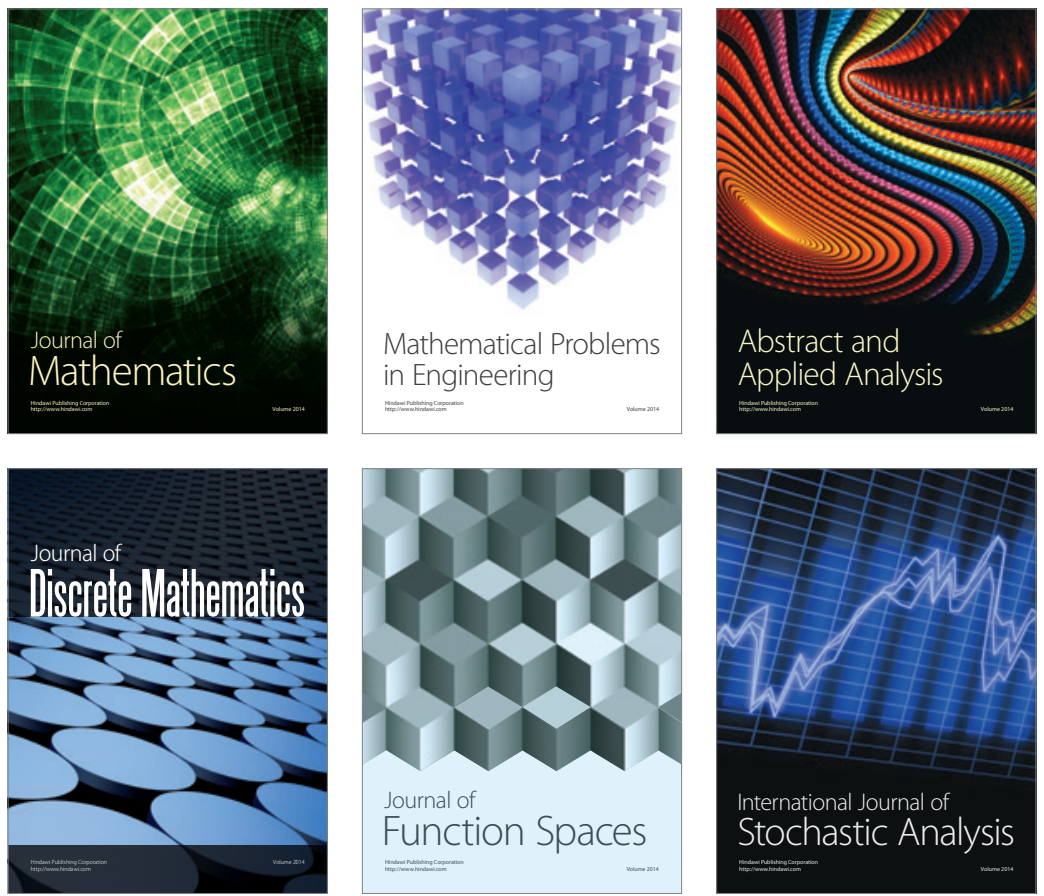

Journal of

Function Spaces

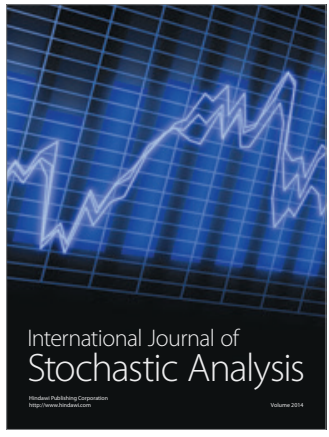

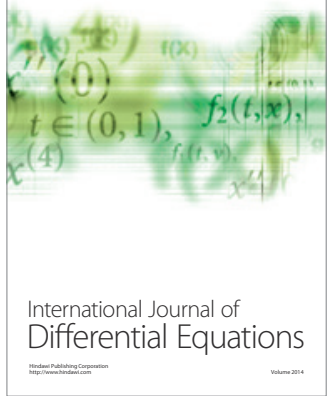
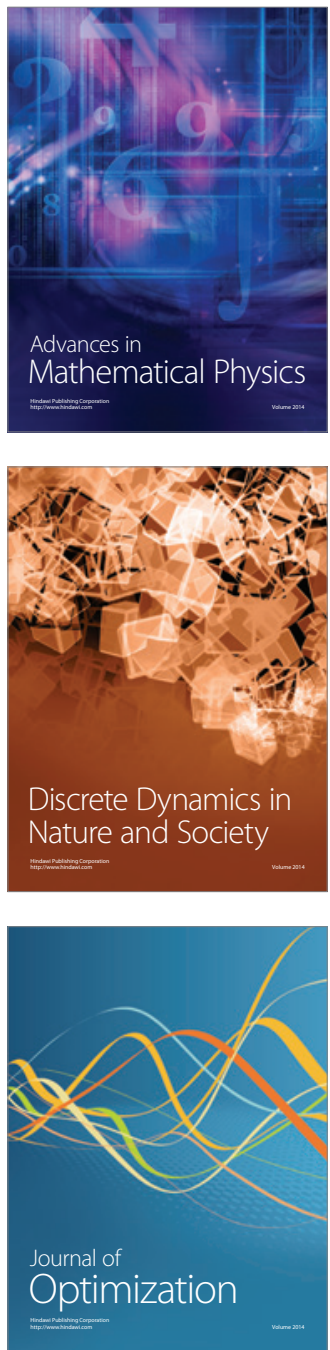